Kansas State University Libraries

New Prairie Press

\title{
THE EFFECT OF MONENSIN ON LACTATION DAIRY COWS: A DOSE RESPONSE EVALUATION
}

Zhanglin Cui

Daniel Mowrey

Alan G. Zimmermann

James T. Symanowski

Howard B. Green

See next page for additional authors

Follow this and additional works at: https://newprairiepress.org/agstatconference

Part of the Agriculture Commons, and the Applied Statistics Commons (c) (i)

This work is licensed under a Creative Commons Attribution-Noncommercial-No Derivative Works 4.0 License.

\section{Recommended Citation}

Cui, Zhanglin; Mowrey, Daniel; Zimmermann, Alan G.; Symanowski, James T.; Green, Howard B.; and Wilkinson, John I. D. (2005). "THE EFFECT OF MONENSIN ON LACTATION DAIRY COWS: A DOSE RESPONSE EVALUATION," Conference on Applied Statistics in Agriculture. https://doi.org/10.4148/ 2475-7772.1138

This is brought to you for free and open access by the Conferences at New Prairie Press. It has been accepted for inclusion in Conference on Applied Statistics in Agriculture by an authorized administrator of New Prairie Press. For more information, please contact cads@k-state.edu. 


\section{Author Information}

Zhanglin Cui, Daniel Mowrey, Alan G. Zimmermann, James T. Symanowski, Howard B. Green, and John I. D. Wilkinson 


\title{
THE EFFECT OF MONENSIN ON LACTATION DAIRY COWS: A DOSE RESPONSE EVALUATION
}

\author{
Zhanglin Cui ${ }^{1}$, Daniel H. Mowrey ${ }^{1}$, Alan G. Zimmermann ${ }^{1}$, James T. Symanowski ${ }^{1}$, \\ Howard B. Green ${ }^{2}$, and John I. D. Wilkinson ${ }^{2}$ \\ ${ }^{1}$ Global Statistics and Information Sciences, Eli Lilly and Company, Indianapolis, IN 46225 \\ ${ }^{2}$ Elanco Animal Health, Eli Lilly and Company, Greenfield, IN 46140
}

\begin{abstract}
Monensin (Rumensin $($ ) was fed at doses of $0,8,16$, or 24 ppm to 966 dairy cows in nine different geographical locations in the USA and Canada. A dose response analysis was conducted on the primary variable, milk production efficiency, to determine the most appropriate dose response function, establish a minimum effective dose, and, when possible, determine a maximum effective dose. Linear mixed models (SAS® Proc Mixed v6.12) were fit to the data. Linear contrasts comparing the non-zero doses of monensin to the control were done to initially determine a minimum effective dose from the 3 non-zero design points. In addition, eight predefined linear contrasts were used to initially determine the general linear-plateau shape of a dose response function for each primary variable. A weighted regression analysis of the least squares means and corresponding standard errors was used when it was necessary to discriminate between the competing linear-plateau functions. A non-overlapping confidence interval process was followed, if it was deemed appropriate, to establish a minimum effective dose for a nondesign point. In cases where the dose response function had a plateau, the dose where the plateau began was classified as the "maximum effective dose" (minimum dose for maximum effect). In cases where the dose response function did not have a plateau, the maximum effective dose was the largest dose used in the study if the response rate was significant.
\end{abstract}

Key Words: monensin, Rumensin, dose response analysis, linear-plateau model, nonoverlapping confidence interval, minimum effective dose, dairy cow.

\section{INTRODUCTION}

To evaluate the effect of monensin (Rumensin ${ }^{\circledR}$ ) (Baile et al., 1982; Meinert et al., 1992; Isch et al., 1999; and Thomas et al., 2004) on lactating dairy cows, nine trials were conducted with six trials sites in the United States (Indiana, North Carolina, Michigan, New York, Florida, and California) and three trial sites in Canada (Ontario, Quebec, and Alberta). Nine hundred sixty-six Holstein dairy cows, including 357 primiparous and 609 multiparous cows, were initially enrolled. A randomized complete block design was used within site and parity. The blocking factors were the anticipated calving date, baseline body weight, and previous lactation milk yield. Within each block, treatments were randomly assigned to animals with treatments being different dose levels of monensin: 0, 8, 16, and 24 parts per million (ppm). 
Data were collected for a total of 57 efficacy variables in the study. Among the 57 variables, 14 were primary variables; and the remaining 43 were secondary variables. The secondary variables were supportive to the primary variables. All the 57 efficacy variables were continuous in nature. Four primary variables were considered for the product label claim (label claim variables).

The purpose of this paper is to present a dose response analysis of the label claim variable, milk production efficiency (Salable 4.0\% Solids Corrected Milk Production Efficiency for the Standardized Lactation Period), to determine the most appropriate dose response function, establish a minimum effective dose, and, when possible, determine a maximum effective dose.

\section{STATISTICAL MODELS AND METHODS}

Two linear mixed models based on restricted maximum likelihood (REML) estimation were used in the data analysis: one for repeated measures and the other for single observation per animal. The two models shared common between-subject fixed factors, a baseline covariate, parity, dose of monensin $(0,8,16$, and $24 \mathrm{ppm})$, and the parity by treatment interaction. The two models differed in the time related fixed and random factors.

\subsection{Model I: Multiple Observations per Animal per Study Period}

For Model I, the repeated measures fixed factor was time (or weeks). Model I is defined as follows:

$$
\mathrm{Y}=\mathrm{X} \beta+\mathrm{Z} v+\varepsilon
$$

where $\mathrm{Y}$ is the response variable, $\mathrm{X} \beta$ and $\mathrm{Zv}$ represent the fixed and random parts of the model, respectively, and $\varepsilon$ is the residual error term, with

$$
\begin{aligned}
& X \beta=\mu+\lambda_{g}\left(X_{g h i j}-\bar{X}_{g}\right)+P_{g}+\tau_{j}+P \tau_{g j}+\alpha_{k}+P \alpha_{g k}+\tau \alpha_{j k}+P \tau \alpha_{g j k}, \text { and } \\
& Z v=\gamma_{h}+\gamma \tau_{h j}+\gamma \beta_{\text {ghi }}+v_{\text {ghij }}
\end{aligned}
$$

In the model described above, $g$ subscripts the parity, $h$ the trial site, $i$ the block level, $j$ the treatment level, and $\mathrm{k}$ the weeks. $X_{\text {ghij }}$ is the baseline value, used as the covariate, and $\bar{X}_{g}$ is the parity-specific mean of the covariate. Construction of the covariate was dependent on the response variable in the analysis. A description of the model terms is as follows: $\mu$ - common mean; $\lambda_{g}$ - slope for regression on covariate; $\mathrm{P}_{\mathrm{g}}$ - parity effect; $\tau_{\mathrm{j}}$ - treatment effect; $\mathrm{P} \tau_{\mathrm{gj}}$ - parity 
by treatment interaction; $\alpha_{\mathrm{k}}$ - relative week effect; $\mathrm{P} \alpha_{\mathrm{gk}}$ - parity by week interaction; $\tau \alpha_{\mathrm{jk}}$ treatment by week interaction; Pt $\alpha_{\mathrm{gjk}}$ - three-factor interaction; $\gamma_{\mathrm{h}}$ - random trial site effect; $\gamma \tau_{\mathrm{hj}}$ site by treatment interaction; $\gamma \beta_{\text {ghi }}$ - block within site effect; and $v_{\text {ghij }}$ - random effect term for animal.

\subsection{Model II: Single Observation per Animal per Study Period}

The following statistical model was used for analysis of variables that were summarized to one value per animal for the study period under consideration.

$$
\mathrm{Y}=\mathrm{X} \beta+\mathrm{Z} v+\varepsilon
$$

where $\mathrm{Y}$ is the response variable, $\mathrm{X} \beta$ and $\mathrm{Z} v$ represent the fixed and random parts of the model, respectively, and $\varepsilon$ is the residual error term, and

$$
\begin{aligned}
& X \beta=\mu+\lambda_{g}\left(X_{\text {ghij }}-\bar{X}_{g}\right)+P_{g}+\tau_{j}+P \tau_{g j}, \text { and } \\
& Z v=\gamma_{h}+\gamma \tau_{h j}+\gamma \beta_{\text {ghi }}
\end{aligned}
$$

The terms and subscripts for Model II are the same as described for Model I.

For all variables, a full model (either Model I or Model II) was firstly fit to test the significance of covariates and covariates by site interactions to determine the inclusion and exclusion of the covariates and interactions in the model. The significance of the interaction of Parity*TRT was used to determine whether the treatment comparison by parity was needed. The significance of the interaction of Site*TRT was used to determine an appropriate denominator degrees of freedom. For the primary variables, heterogeneity of parity and site was tested using Levene's (1960) test to determine whether a weighted analysis was needed. Based on the above tests, a final model was then determined. Treatment contrasts were constructed and least-squares means and associated standard error were estimated in the final model for all variables.

For the label claim variables, a dose response analysis was performed. The rest of this paper will focus on the dose response analysis for the label claim variables. As an example, a dose response analysis will be discussed in detail for the claim variable "salable $4.0 \%$ solids corrected milk production efficiency for the standardized lactation period”.

\section{DOSE RESPONSE ANALYSIS}

Data from the nine trials for a label claim variable were pooled and analyzed using Model I or Model II to construct treatment contrasts, estimate the overall residual of the model, and estimate 
least-squares means and associated standard errors for the treatment groups. A dose response analysis was further conducted to determine the most appropriate dose response function, establish a minimum effective dose, and determine a maximum effective dose.

\subsection{Establishing a Dose Response Curve}

A dose response function was established for the label claim variable. From the models that were fit to the data for the claim variable, linear contrasts were used to initially determine the general shape of the dose response function. Eight single degree of freedom contrasts were proposed before examining the data (Table 1). These contrasts included most of the shapes described by Anderson and Nelson (1975).

Table 1. Treatment Contrasts for Evaluation of Dose Response

Treatment

Contrasts

\begin{tabular}{lrr}
\multicolumn{4}{c}{ Monensin Dose $(\mathrm{ppm})$} \\
\hline 0 & 8 & 16
\end{tabular}

Description

\begin{tabular}{rrrrll}
\hline 1 & -3 & -1 & 1 & 3 & Linear from control \\
2 & -3 & -3 & 1 & 5 & Linear from 8 ppm \\
3 & -2 & 0 & 1 & 1 & Changing slopes I \\
4 & -9 & -1 & 3 & 7 & Changing slopes II \\
5 & -3 & 1 & 1 & 1 & Plateau at 8 ppm \\
6 & -1 & -1 & 1 & 1 & Threshold at 8 ppm, plateau at 16 ppm \\
7 & -5 & -1 & 3 & 3 & Linear from control, plateau at 16 ppm \\
8 & -1 & 0 & 1 & 0 & Peak at 16 ppm
\end{tabular}

The contrast with the smallest p-value or largest t-statistic was considered the "best" description of the response for a particular claim variable.

When two or more treatment contrasts resulted in p-values of similar magnitude, further discrimination among the corresponding response functions was necessary. A weighted regression analysis of the least squares means and corresponding standard errors was then conducted to aid in the discrimination of the different response functions. The response variable was the treatment group least squares means and the weighting variable was the squared reciprocal of the corresponding standard errors, $\left(1 /\right.$ stderr $^{2}$. The regression analysis of least squares means used concepts described by Anderson and Nelson (1975). However, the design matrices for the response functions for the claim variables were different than the matrices used by Anderson and Nelson. A variable TRT was designated to reflect the actual monensin doses $(0,8,16$, or $24 \mathrm{ppm})$, which implied a straight regression line. Two other variables, TRT2 and TRT_3, were used to modify the fit to various curves. A more detailed discussion of the design matrices will be given in the discussion that follows. 


\subsection{Establishing a Minimum Effective Dose}

For each of the label claim variables, a mixed model analysis was conducted in which linear contrasts were used to compare the non-zero doses of monensin used in the study to the controls. Two-tailed tests were conducted for the linear contrasts and the significance level for the tests was 0.05. A minimum effective dose was established for each of the label claim variables by determining the lowest dose that was different $(\mathrm{P}<0.05)$ from the controls. The establishing of a minimum effective dose using the linear contrast technique could only be used for the design points of the study.

In addition to the linear contrast process to establish a minimum effective dose for a design point, a non-overlapping confidence interval process was followed to establish a minimum effective dose for a non-design point for the four claim variables. The non-overlapping confidence interval process used a linear regression on the least squares means to generate estimates to be used in constructing the confidence limits. The generation of the $95 \%$ confidence limits was done using the following formula:

$$
\mathrm{X}_{0} \mathrm{~b} \pm \mathrm{t}_{(v, 0.975)} \times \mathrm{s} \sqrt{\mathrm{X}_{0}\left(\mathrm{X}^{\prime} \mathrm{X}\right)^{-1} \mathrm{X}_{0}^{\prime}}
$$

The value $X_{0}$ is a 1 by 2 vector $(1, M)$, where $M$ is a dose of monensin. The value $b$ in the formula is a 2 by 1 vector containing the estimated intercept and slope from the regression line. The value of $\mathrm{t}_{(v, 0.975)}$ is a two-tailed $\mathrm{t}$-statistic with degrees of freedom, $v$, and significance level, 0.95. The value of $\mathrm{s}$ is the square root of the mean square error from the regression and $\mathrm{X}$ is the model matrix used in the regression.

In using the non-overlapping confidence interval technique, 95\% confidence intervals were constructed about each non-zero dose of monensin in the dose response range, with vector $\mathrm{X}_{0}=$ $(1, M)$, where $M$ was a number greater than zero representing a dose of monensin. A 95\% confidence interval was also constructed about the 0 dose, with vector $\mathrm{X}_{0}=(1,0)$. Assuming the slope $\beta>0$, the minimum effective dose for each claim variable was defined as the lowest dose $(\mathrm{M})$ in which lower limit (upper limit if slope $\beta<0$ ) of its $95 \%$ confidence interval did not overlap with the upper limit (lower limit if slope $\beta<0$ ) of the $95 \%$ confidence interval for the 0 dose.

To preserve the results from the mixed model and satisfy the assumptions of the simple regression, a hybrid approach to the non-overlapping confidence interval technique was taken for all four claim variables.

The hybrid approach utilized the model fit from the weighted regression to determine $b$ in the above formula, but utilized variance components from the mixed model to determine $\mathrm{s}$ in the above formula. For each of the four claim variables, the Site by Treatment variance component 
was estimated as zero, and therefore, was not included in the variance for the non-overlapping confidence interval technique. The square root of the mean square error (MSE) was determined from the mixed model analysis, and used for $\mathrm{s}$ in the above formula. The $\mathrm{X}^{\prime} \mathrm{X}$ matrix was calculated based on the actual treatment group sample sizes from the mixed model analysis. See details in the following sections.

\subsection{Establishing a Maximum Effective Dose}

For each claim variable, the "best" response function was determined from the eight pre-defined linear contrasts in conjunction with a weighted regression analysis. In those cases where the dose response function had a plateau, the dose where the plateau began was classified as the "maximum effective dose" (minimum dose for maximum effect). In cases where the dose response function did not have a plateau, the maximum effective dose was the largest dose used in the study.

\section{A CASE STUDY: MILK PRODUCTION EFFICIENCY}

\subsection{Establishing a Dose Response Curve}

A linear mixed model (Model II) was used to analyze the label claim variable "salable $4.0 \%$ solids corrected milk production efficiency for the standardized lactation period". The variable name "salable $4.0 \%$ solids corrected milk production efficiency for the standardized lactation period" is referred to as "milk production efficiency" throughout the remainder of this paper. The mean efficiencies increased approximately linearly with increasing doses of monensin. Among the eight competing treatment contrasts testing various shapes of dose response curves, three contrasts were candidates based on the criteria selected: linear from control $(\mathrm{t}=3.73, \mathrm{P}$ $=0.0002)$, changing slopes II $(\mathrm{t}=3.62, \mathrm{P}=0.0003)$, and linear from $8 \mathrm{ppm}(\mathrm{t}=3.62, \mathrm{P}=0.0003)$. To further discriminate among these three dose response shapes, weighted least squares regressions of the treatment group least squares means on the monensin doses were conducted.

In the changing slopes II model, one slope was fit for doses between 0 and $8 \mathrm{ppm}$, and a different slope was fit for doses between 8 and $24 \mathrm{ppm}$. In the linear from $8 \mathrm{ppm}$ model, a slope of zero was imposed for doses between 0 and $8 \mathrm{ppm}$, and a non-zero slope was fit for doses between 8 and $24 \mathrm{ppm}$. A linear from control model was fit with a single slope for doses from 0 to 24 ppm. These models were fit using SAS Proc REG after defining the following SAS variables (design matrix):

$\begin{array}{ll}\text { TRT } & \text { TRT2 } \\ 0 & 8 \\ 8 & 0 \\ 16 & 0 \\ 24 & 0\end{array}$


The procedure REG was invoked with the following SAS code:

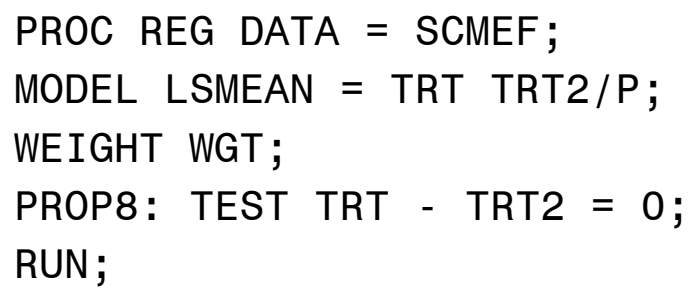

The model in the above codes was expressed as a linear regression equation:

$$
\mathrm{E}(\mathrm{Y})=\alpha+\beta^{*} \mathrm{TRT}+\beta_{2} * \mathrm{TRT} 2
$$

Substituting the values defined in the design matrix for the variables TRT and TRT2, expected means were obtained:

$$
\begin{aligned}
& \mathrm{E}\left(\mathrm{Y}_{0}\right)=\alpha+8 \beta_{2} \\
& \mathrm{E}\left(\mathrm{Y}_{8}\right)=\alpha+8 \beta \\
& \mathrm{E}\left(\mathrm{Y}_{16}\right)=\alpha+16 \beta \\
& \mathrm{E}\left(\mathrm{Y}_{24}\right)=\alpha+24 \beta
\end{aligned}
$$

If $\beta_{2}=0$, the dose response becomes a linear from $0 \mathrm{ppm}$ model. If $\beta_{2} \neq 0$ and $\beta_{2}=\beta$, the dose response becomes a linear from $8 \mathrm{ppm}$ model. Finally, if $\beta_{2} \neq 0$ and $\beta_{2} \neq \beta$, the dose response is a changing slopes II model.

Testing whether the coefficient associated with TRT2 was zero or not determined whether the dose response was a linear from control model or one of the other two models. The p-value for $\beta_{2}$ was 0.1433 , indicating a zero coefficient associated with TRT2. Therefore, the linear from control model was not rejected. Since $\beta_{2}=0$ was not rejected, no further test was needed. Thus, neither changing slopes II model nor linear from $8 \mathrm{ppm}$ model was superior to the linear from control model. It was concluded that the best model describing the effect of monensin dose on milk production efficiency was linear from control.

The linear from control model was fit using the SAS procedure REG after defining the following SAS variable:

TRT
0
8
16
24


The procedure REG was invoked with the following SAS code:

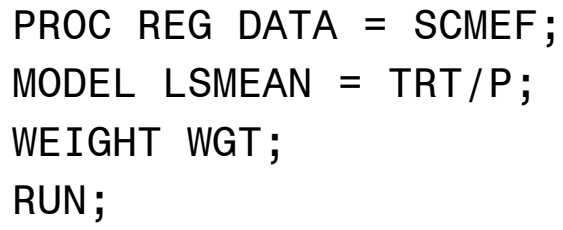

The linear from control model (Figure 1) is represented by the following simple linear regression, including the parameter estimates for the slope and intercept:

$$
\mathrm{E}(\mathrm{Y})=0.8211+(0.0014) \mathrm{M}
$$

where $\mathrm{M}$ in place of TRT represents the dose of monensin (ppm) and $\mathrm{E}(\mathrm{Y})$ the mean for milk production efficiency associated with $\mathrm{M}$.

\subsection{Establishing a Minimum Effective Dose}

Treatment comparisons in the mixed model analysis (Model II) revealed that 16 and $24 \mathrm{ppm}$ groups were different from control $(\mathrm{P} \leq 0.0231)$. This defined the minimum effective dose of 16 ppm as a design dose point. However, it is necessary to determine if a non-design dose point existed that could be classified as a minimum effective dose. A non-overlapping confidence interval technique was conducted to identify the minimum effective dose of monensin at a nondesigned dose level.

Upon conducting a mixed model analysis (Model II) for the individual animal milk production efficiency data, the square root of the mean square error was determined, (0.0078), as the most appropriate values to be used in the formula for the $95 \%$ confidence limit. The degrees of freedom associated with the mean square error from Model II (807) were used to determine the value of the t-statistic (1.96). The $X^{\prime} X$ matrix was calculated based on the actual treatment group sample sizes from the mixed model analysis of milk production efficiency $(200,204,211$, and 200 animals for the $0,8,16$, and 24 ppm groups, respectively). The intercept and slope used in the calculation of the confidence intervals were those estimated from the regression on the least squares means. The following equations were used to calculate $95 \%$ confidence intervals for the mean milk production efficiency at a dose of monensin equal to $\mathrm{M}$ :

$$
(1, \mathrm{M})\left(\begin{array}{l}
0.8211 \\
0.0014
\end{array}\right) \pm 1.96 \times \sqrt{0.0078 \times(1, \mathrm{M})\left(\left(\begin{array}{cc}
815 & 9808 \\
9808 & 182272
\end{array}\right)^{-1}\right)\left(\begin{array}{c}
1 \\
\mathrm{M}
\end{array}\right)}
$$

when $0 \leq \mathrm{M} \leq 24$. 
The upper limit of the confidence interval for $0 \mathrm{ppm}$ was $0.8312 \mathrm{~kg} / \mathrm{Mcal}$. The lower limits of the confidence intervals for 11,12 , and $13 \mathrm{ppm}$ were $0.8300,0.8314$, and 0.8327 respectively. The first non-zero dose for which the lower confidence limit did not overlap with the upper confidence limit of the control was $12 \mathrm{ppm}$. Upon examining Figure 1, it can be seen that the horizontal line across the graph at the upper limit of the confidence interval for the 0 ppm group intersects the plot of the lower confidence interval at about $12 \mathrm{ppm}$. Therefore, according to the non-overlapping confidence interval technique, the minimum effective dose of monensin was calculated to be $12 \mathrm{ppm}$.

\subsection{Establishing a Maximum Effective Dose}

Since the most appropriate model for the data was a linear from control model, the maximum effective dose was the largest design point, 24 ppm.

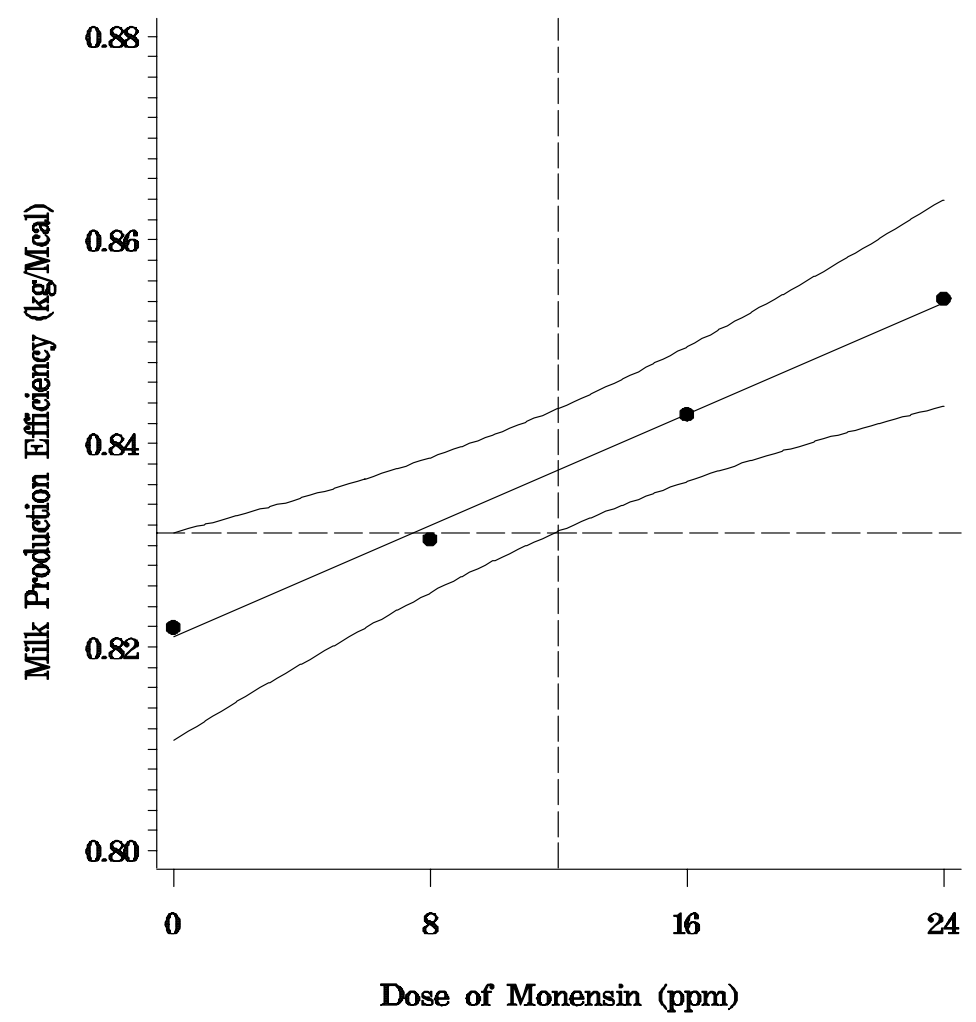

Linear from Control Model with 95\% Confidence Interval

Figure 1. Milk Production Efficiency 


\subsection{Summary}

Milk production efficiency increased linearly with increasing doses of monensin throughout the entire dose range of 0 to $24 \mathrm{ppm}$ at a rate of $1.4 \mathrm{~g}$ of salable $4.0 \%$ solids corrected milk per Mcal of $\mathrm{NE}_{\mathrm{L}}$ intake per ppm of monensin. Data from the clinical field trials supported that monensin fed in the range of 12 to $24 \mathrm{ppm}$ increases $(\mathrm{P} \leq 0.05)$ milk production efficiency.

\section{REFERENCES}

Anderson, R.L., and Nelson, L.A. 1975. A Family of Models Involving Intersecting Straight Lines and Concomitant Experimental Designs Useful in Evaluating Response to Fertilizer Nutrients. Biometrics 31, pp. 303-318.

Baile, C.A., C.L. McLaughlin, W.V. Chalupa, D.L. Snyder, L.C. Pendlum, and E.L. Potter. 1982. Effects of monensin fed to replacement dairy heifers during the growing and gestation period upon growth, reproduction and subsequent lactation. J. Dairy Sci. 65:1941-1944.

Isch, J.A., J.E. Shirley, M.V. Scheffel, E.C. Titgenmeyer and E.C. Thomas. 1999. Effects of Rumensin and Bovatec ${ }^{\circledR}$ on growth, feed intake and feed efficiency in dairy calves. 1999 Dairy Day Report of Progress 842, Kansas State University. Bovatec ${ }^{\circledR}$ is a registered trademark of Alpharma Inc..

Levene, H. 1960. Contributions to Probability and Statistics. Stanford University Press, Stanford, CA, p. 278.

Meinert, R.A., C.M.J. Young, A.J. Heinrichs and G.A. Varga. 1992. Effect of monensin on growth, growth estimated body composition in Holstein heifers. J. Dairy Sci. 75:257-261.

Thomas, Elvin E., Howard B. Green, David G. McClary, John I. D. Wilkinson, R. Ken McGuffey, Angel A. Aguilar, Gerald D. Mechor, and Zhangin Cui. 2004. Effect of Rumensin ${ }^{\circledR}$ (monensin sodium) on Performance Parameters of Lactating Dairy Cows - Nine-trial Registration Summary. Tech Talk Scientific Update from Elanco Animal Health. Available at http://www.elancous.com/docs/rumensin/dairytools/EAH3027_9TrialEffTT.pdf 